\title{
Towards Quality-of-Service Control of Networked Control Systems: A Switched Time Delay Systems Approach
}

\author{
Chih-Chung Chen, Sandra Hirche* and Martin Buss
}

\begin{abstract}
The stability and performance of a networked control system (NCS) strongly depends on the communication quality in terms of time delay for example. According to the Quality-of-Service (QoS) concept in modern communication technology, the communication quality can be adapted to the requirements of the network application. This paper presents a first approach to conjointly control the NCS as well as the communication quality on basis of the QoS concept. We assume that the controller together with the time delay is switched to meet control and network performance objectives. Sufficient stability conditions are presented for the resulting switched time delay system based on the concept of piecewise continuous Lyapunov functions and the Razumikhin approach. Simulations and experiments validate the proposed approach.
\end{abstract}

\section{INTRODUCTION}

In the view of affordability, widespread usage and well developed infrastructure, communication networks are very attractive for the signal transmission in control systems. This comes, however, at the cost of a no longer ideal signal transmission. The intrinsic communication unreliabilities, such as (varying) time delay and packet loss, have a strong influence on the stability and the performance of the closed loop system. In the current NCS literature the communication quality is assumed to be given in advance, accordingly stabilizing controllers are designed. Differently, in this paper the communication quality is considered to be controllable. This is motivated by the Quality-of-Service (QoS) concept in modern communication technology. Generally, QoS refers to the capability of a network to provide different communication quality to different network traffic. The communication quality can be adapted to the requirements of the network application. Depending on the QoS network architecture deterministic bounds on time delay and packet loss can be guaranteed, e.g. with the Integrated Service architecture [1]. A first implementation of the QoS concept can be found in the Internet protocol IPv6.

Inspired by the QoS concept an innovative control architecture is investigated here: It combines the control of the time delay and the appropriate change of the system controller following stability and performance requirements. Here the time delay guaranteed by QoS is assumed to be

Institute of Automatic Control Engineering, Technische Universität München, D-80290 München, Germany; http : //www.lsr.ei.tum.de, chen@1sr.ei.tum.de,m.buss@ieee.org

*S. Hirche is a visiting researcher with Fujita Lab., Dept. of Mechanical and Control Engineering, Tokyo Institute of Technology, 152-8552 Tokyo, Japan; s.hirche@ieee.org

This work was supported in part by the Japanese Society for the Promotion of Science (JSPS) by a Postdoctoral Fellowship for Foreign Researchers granted to the second author.

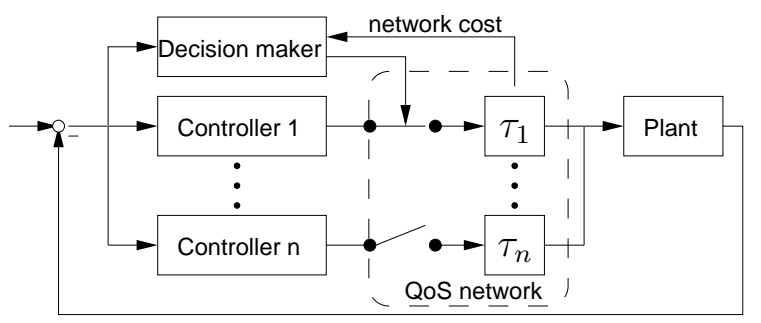

Fig. 1. Architecture of the QoS controlled NCS: A switched time delay system.

switchable between different levels resulting in piecewise constant time delay. The controller is synchronously adapted, see Fig. 1 for a visualization of the principal QoS control architecture. Aiming at maximizing the control performance, generally, low time delay is desirable. However, low time delay (high network quality) requires large network resources, i.e. induces high network cost. Considering the finite network resources it is desirable that each network application only consumes as much of them as required to guarantee the desired level of control performance.

In this paper the time delay as well as the controller are switched. Switched (hybrid) systems are dynamical systems that consist of a finite set of subsystems and a logical rule that orchestrates the switchings between them. The stability of the subsystems themselves is not sufficient for the stability of the overall system [2], [3]. Stability with arbitrary switching can be ensured by the existence of a common Lyapunov function. However, it is usually difficult to find a common Lyapunov function for all candidates subsystems. As an alternative, the concept of piecewise continuous Lyapunov functions is developed in [4]-[6]. Dwell-time based switching is considered in [7], [8]. These approaches, however, do not consider time delay. Most prominent for the analysis of time delay systems are Lyapunov-like approaches based either on the Razumikhin or the Krasovskii method, see e.g. [9][12] and references therein. Hybrid time delay systems are considered in [13], [14] using an extension of the common Lyapunov function approach, and adopting a dwell-time based switching approach [15]. However, the time delay itself is not switched in these works.

To the best knowledge of the authors for the first time a switched system with switched time delays is considered in this paper. The stability is analyzed by the construction of piecewise continuous Lyapunov-Razumikhin functions, and a sufficient stability condition is derived. The potential of the proposed QoS control approach is examined in simulations 
and experiments. Therefore for each considered time delay a controller is designed using a standard LMI method for delay-dependent controller design. The optimal switching instants are determined by numerical minimization of a cost function which comprises the control performance and the network cost.

The remainder of the paper is organized as follows: The problem setting is stated in Section II. Section III introduces the foundations of stability analysis for both time delay and switched systems. In Section IV, the main result on the stability of switched time delay system is presented. The optimal performance is discussed in Section V; the approach is validated in simulations and experiments.

\section{PRoblem Formulation}

The general architecture of the QoS controlled NCS considered in this paper is illustrated in Fig. 1 The network induced time delay as well as the controller are synchronously adjusted by a decision-maker. The generation of the switching law is discussed in Section V. The switching mechanism causes the variation of the system dynamics and results in a switched time delay system. In the general case of nonlinear subsystems, the overall system dynamics is described by a delay differential functional of the form

$$
\dot{x}(t)=f_{\sigma(t)}\left(x(t), x_{t}, \tau_{\sigma(t)}\right),
$$

where $x \in \mathbb{R}^{n}$ is the state, having the same dimension for all subsystems, and $\tau_{\sigma(t)} \in \mathbb{R}^{+}$represents the piecewise constant time delay. The switching signal $\sigma(t)$ is a piecewise constant finite valued function taking values on the set $\mathcal{P}:=\{1, \ldots, N\}$. If $\sigma(t)=i$, then subsystem $i$ is active in the time interval $t \in\left[t_{j}, t_{j+1}\right) ; N$ represents the number of subsystems. By $x_{t} \in \mathbb{R}^{n}$ the initial condition of the time delay functional is specified which has the form of

$$
x_{t}=x(t+\theta), \theta \in\left[-\tau_{\sigma(t)}, 0\right],
$$

hence at each switching instant $t$ the "length" of the initial condition depends on the time delay of the previous active subsystem. A finite number of switches is assumed in finite time. The problem is assumed to be well-posed in the sense that for each initial condition and switching sequence there exists a unique solution. A simplified model is considered in this first analysis with the forward and backward time delays lumped into the single value $\tau_{\sigma(t)}$. Note that this assumption represents no restriction in case of linear subsystems or control affine nonlinear subsystems with proportional state feedback, and with synchronous switching of forward and backward delay.

In contrast to conventional switched systems, the proposed control structure comprises switching of controllers and time delays. To the best knowledge of the author, there exists no appropriate analysis and design method for such system until now. The stability analysis starts from time delay systems and extends to switched time delay systems. The background on stability of switched systems and time delay systems is introduced in the following.

\section{FundAMENTALS}

\section{A. Stability of Switched Systems}

Switching between stable subsystems may result in instability of the overall system [2], [3]. Stability with arbitrary switching can be ensured by the existence of a common Lyapunov function. As a common Lyapunov function is generally difficult to find, an approach based on piecewise continuous Lyapunov functions is considered in this paper. Piecewise continuous Lyapunov functions are first introduced for linear switched systems in [4], extended to nonlinear switched systems in [5]; a very general result is derived in [6].

Consider the switched system (1) without time delay

$$
\dot{x}(t)=f_{\sigma(t)}(x(t)) .
$$

Consider a Lyapunov function associated to each subsystem. Then the stability of the switched system (3) is ensured by a nonincreasing sequence of the Lyapunov function values at the switching instants into each subsystem. The precise statement is given as follows.

Theorem 1: Given a switched (nonlinear) system (3) with a Lyapunov function $V_{i}$ with the equilibrium point at $x=0$ associated to each subsystem $f_{i}, i \in \mathcal{P}$. Let $t_{k}>t_{j}$ be switching times for which $\sigma\left(t_{k}\right)=\sigma\left(t_{j}\right)=i$ such that

(i) $V_{i}(t, 0)=0$ and $V_{i}(t, x)>0$ for $x \in \mathbb{R}^{n} \backslash\{0\}$,

(ii) $V_{i}(t, x(t)) \leq \gamma\left(V_{i}\left(t_{j}, x\left(t_{j}\right)\right)\right), \forall t \in\left[t_{j}, t_{j+1}\right)$ with $\gamma(\cdot)$ a positive continuous function and $\gamma(0)=0$,

(iii) $V_{i}\left(t_{k}, x\left(t_{k}\right)\right)-V_{i}\left(t_{j}, x\left(t_{j}\right)\right) \leq-h\left(\left\|x\left(t_{j}\right)\right\|\right)$, where $\|\cdot\|$ denotes Euclidean norm, $h(\cdot)$ is a positive continuous function and $h(0)=0$.

Then the switched system (3) is Lyapunov stable. A strictly decreasing sequence at the consecutive switching instants for which $\sigma\left(t_{k}\right)=\sigma\left(t_{j}\right)$ implies asymptotic stability.

Proof: See [6].

The stability condition of Theorem 1 is illustrated in Fig. 2. The values of the corresponding Lyapunov function at the switching instants are marked by filled symbols. The switched system is asymptotically stable due to the strictly decreasing values of Lyapunov functions at consecutive switching instants.

\section{B. Stability of Time Delay Systems}

The standard time delay system is recovered from (1) by assuming no switching occurs, i.e. $\sigma(t)=$ const. Two different techniques are commonly used for the stability analysis of time delay systems; the Lyapunov-Krasovskii approach and the Lyapunov-Razumikhin approach, see e.g. [9]-[12]. The construction of the Lyapunov function by the Krasovskii approach results in a functional $V\left(t, x_{t}\right)$ depending on the delayed state (initial condition) $x_{t}$, which is rather difficult to analyze. The Razumikhin approach circumvents the difficulties by considering the Lyapunov function $V(t, x(t))$ depending on the present state $x(t)$ only. Asymptotic stability is guaranteed if the Lyapunov function $\xi V(t, x(t)), \xi>1$, 


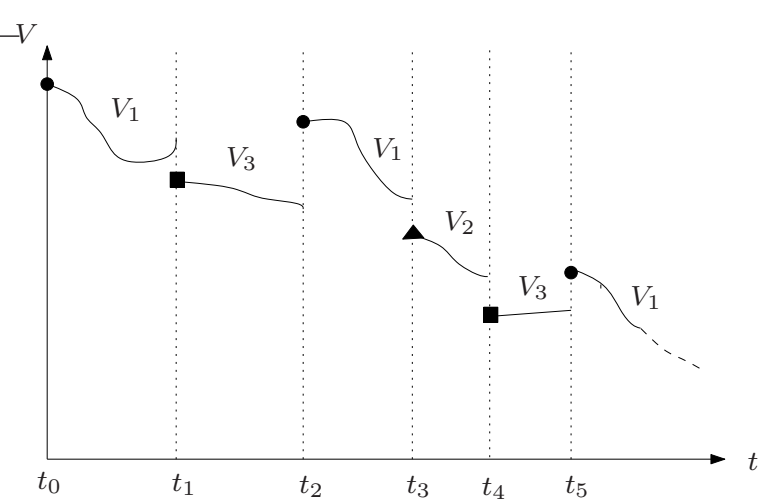

Fig. 2. Piecewise continuous Lyapunov function of a switched system with 3 subsystems, the values at the switching instants in a certain subsystem form a strictly decreasing sequence.

does not exceed the maximum value

$$
\bar{V}\left(t, x_{t}\right)=\max _{\theta \in[-\tau, 0]} V(t+\theta, x(t+\theta)),
$$

of the Lyapunov function over the past time delay interval as stated more precisely in the following.

Theorem 2: (Razumikhin Theorem) A time delay system (1) with $\sigma(t)=$ const. is said to be asymptotically stable if there exists a continuous differentiable positive definite function $V: \mathbb{R}^{n} \rightarrow \mathbb{R}^{+}$such that

(i) $u_{1}(\|x(t)\|) \leq V(t, x(t)) \leq u_{2}(\|x(t)\|)$ for some continuous nondecreasing function $u_{1}$ and $u_{2}, u_{1}(s)>0$, $u_{2}(s)>0$ for $s>0$ and $u_{1}(0)=0=u_{2}(0)$,

(ii) $\dot{V}(t, x(t))<-w(\|x(t)\|)$, whenever $V(t+\theta, x(t+\theta)) \leq p(V(t, x(t)))$ for all $\theta \in[-\tau, 0]$. Where $w(s)$ and $p(s)$ are continuous nondecreasing functions $w(s)>0, p(s)>0$ for $s>0$.

Proof: See [11].

In the performance evaluation later in this paper, we consider a switched time delay system with linear time invariant (LTI) subsystems

$$
\begin{aligned}
\dot{x}(t) & =A_{0} x(t)+A_{1} x(t-\tau), \\
x_{t} & =x(t+\theta), \forall \theta \in[-\tau, 0],
\end{aligned}
$$

with the system matrices $A_{0}, A_{1} \in \mathbb{R}^{n \times n}$, and the subsystem index $i \in \mathcal{P}$ omitted here for simplicity. Using a quadratic Lyapunov function with the Razumikhin approach, a delaydependent stability criterion can be formulated as a LMI problem as presented in the following corollary.

Corollary 1: The time delay system(5) is asymptotically stable if there exists real symmetric matrix $P>0$ and real scalars $\alpha>0, \alpha_{1}>0$ such that

$$
\left(\begin{array}{ccc}
M & -P A_{1} A_{0} & -P A_{1}^{2} \\
-A_{1}^{T} A_{0}^{T} P & -\alpha P & 0 \\
-\left(A_{1}^{2}\right)^{T} P & 0 & \alpha_{1} P
\end{array}\right)<0,
$$

where $M=\frac{1}{\tau}\left[P\left(A_{1}+A_{0}\right)+\left(A_{1}+A_{0}\right)^{T} P\right]+\left(\alpha+\alpha_{1}\right) P$. Proof: See [12].

Based on these approaches for switched systems and time delay systems we will derive our main result in the following.

\section{Main Result: Stability of Switched Time DELAY SYSTEMS}

We assume a switched time delay system given by (1) with asymptotically stable subsystems $f_{i}$. A continuously differentiable Lyapunov function $V_{i}$ is associated to each subsystem $i$, where in general $V_{i}(x) \neq V_{j}(x)$ holds if $i \neq j$. Furthermore, suppose that there is no state jump in the state of (1) at the switching instants, i.e. the solution $x(t)$ is continuous everywhere.

The following theorem extends the known Razumikhin theorem to switched systems with piecewise continuous time delays and providing sufficient conditions for asymptotic stability.

Theorem 3: A switched time delay system (1) is said to be asymptotically stable if there exists a continuously differentiable positive definite function $V_{i}$ for each subsystem $i \in \mathcal{P}$, active in the time interval $t \in\left[t_{j}, t_{j+1}\right)$, such that

(i) $V_{i}(t, 0)=0, V_{i}(t, x(t))>0$ for $x \in \mathbb{R}^{n} \backslash\{0\}$,

(ii) $u_{1}(\|x(t)\|) \leq V_{i}(t, x(t)) \leq u_{2}(\|x(t)\|)$, where $u_{1}, u_{2}$ are continuous nondecreasing functions $u_{1}(s)>0$, $u_{2}(s)>0$ for $s>0$ and $u_{1}(0)=0=u_{2}(0)$,

(iii) $\dot{V}_{i}(t, x(t)) \leq-w(\|x(t)\|), \quad$ whenever $V_{i}(t+\theta, x(t+\theta)) \leq p\left(V_{i}(t, x(t))\right)$ with $\theta \in\left[-\tau_{i}, 0\right]$, $t \in\left[t_{j}, t_{j+1}\right) . \quad w(s)$ and $p(s)$ are continuous nondecreasing functions $w(s)>0, p(s)>0$ for $s>0$ and $\dot{V}_{i}(t, x(t))$ is the right-hand derivative $\left.\dot{V}_{i}(t, x(t))=\lim _{\Delta \rightarrow 0, \Delta>0} \frac{V_{i}(t+\Delta, x(t+\Delta))-V_{i}(t, x(t))}{\Delta}\right)$,

(iv) $\bar{V}_{i}\left(t_{k}, x_{t_{k}}\right)-\bar{V}_{i}\left(t_{j}, x_{t_{j}}\right) \leq-h\left(\left\|x_{t_{j}}\right\|\right), \quad h(\cdot)$ is a positive continuous function with $h(0)=0$ and $t_{k}>t_{j}$ are consecutive switching instants for which $\sigma\left(t_{k}\right)=\sigma\left(t_{j}\right)=i$.

Proof: For the sake of simplicity, the switched time delay system (1) is assumed to contain two subsystems $\dot{x}=f_{1}\left(x, x_{t}, \tau_{1}\right)$ and $\dot{x}=f_{2}\left(x, x_{t}, \tau_{2}\right)$. Without loss of generality, the switched time delay system starts in subsystem 1 at $t_{0}$ with the initial condition $x_{t_{0}}=x\left(t_{0}+\theta\right)$, $\theta \in\left[-\tau_{1}, 0\right]$ assuming $\left\|x_{t_{0}}\right\|<\delta_{1}$. The associated Lyapunov function is $V_{1}$. By (ii), there exists an $\varepsilon_{1}>u_{1}^{-1}\left(u_{2}\left(\delta_{1}\right)\right)>0$ such that $\bar{V}_{1}\left(t_{0}, x_{t_{0}}\right) \leq u_{2}\left(\delta_{1}\right)<u_{1}\left(\varepsilon_{1}\right)$. Since subsystem 1 is asymptotically stable by (i)-(iii), the Lyapunov function satisfies $V_{1}(t, x(t))<\bar{V}_{1}\left(t_{0}, x_{t_{0}}\right)<u_{1}\left(\varepsilon_{1}\right)$ for all $t$ with $t_{0} \leq t<t_{1}$, where $t_{1}$ marks the switching instant when subsystem 1 is switched into subsystem 2. In addition, $\lim _{t_{1} \rightarrow \infty}\left\|x\left(t_{1}\right)\right\|=0$ following from (iii).

At the time instant $t_{1}$ the execution enters subsystem 2 , where $t_{1} \geq t_{0}+\max _{\{1,2\}}\left(\tau_{i}\right)-\tau_{1}$ is required for the proper definition of the initial condition for subsystem 2 . Since there is no jump in the states at the switching instant, the initial condition for subsystem 2 is given by $x_{t_{1}}=x\left(t_{1}+\theta\right)$ with $\theta \in\left[-\tau_{2}, 0\right]$. By (iii) again, the associated Lyapunov function $V_{2}(t, x(t))$ can never exceed $\bar{V}_{2}\left(t_{1}, x_{t_{1}}\right), \forall t: t_{1} \leq t<t_{2}$ where $t_{2}$ marks the switching instant from subsystem 2 back to subsystem 1 . Since $\left\|x_{t_{1}}\right\|<\varepsilon_{1}$, there is an $\varepsilon_{2}>0$ such that $u_{2}^{-1}\left(u_{1}\left(\varepsilon_{2}\right)>\varepsilon_{1}\right.$. With the same argument as above it follows that $\|x(t)\|<\varepsilon_{2}$, for all $t$ with $t_{1} \leq t<t_{2}$. Furthermore, it follows by (iii) $\lim _{t_{2} \rightarrow \infty}\left\|x\left(t_{2}\right)\right\|=0$. 
At the time instant $t_{2}$, the execution re-enters subsystem 1 . By (iv) $\quad \bar{V}_{1}\left(t_{2}, x_{t_{2}}\right) \leq \bar{V}_{1}\left(t_{0}, x_{t_{0}}\right)-h\left(\left\|x_{t_{0}}\right\|\right)<u_{1}\left(\varepsilon_{1}\right)$ holds, hence $\|x(t)\|<\varepsilon_{1}$ for all $t$ with $t_{2} \leq t<t_{3}, t_{3}$ the next time instant when subsystem 1 switched back to subsystem 2 . With the same argument, it can be shown that $\bar{V}_{2}\left(t_{3}, x_{t_{3}}\right)<u_{1}\left(\varepsilon_{2}\right)$.

At each switching instant $t_{j}$, the Lyapunov function $\bar{V}_{\sigma\left(t_{j}\right)}\left(t_{j}, x_{t_{j}}\right)$ is bounded from above by $u_{1}\left(\varepsilon_{2}\right)$, which implies the existence of a $z \geq 0$ defined as $z=\lim _{j \rightarrow \infty} \bar{V}_{\sigma\left(t_{j}\right)}\left(t_{j}, x\left(t_{j}\right)\right)$. As a result for each subsystem $i$ and any two consecutive switching instants $t_{k}>t_{j}$, $\sigma\left(t_{k}\right)=\sigma\left(t_{j}\right)=i$, by (iv) the following holds

$$
\begin{aligned}
0 & =\lim _{k \rightarrow \infty} \bar{V}_{i}\left(t_{k}, x\left(t_{k}\right)\right)-\lim _{j \rightarrow \infty} \bar{V}_{i}\left(t_{j}, x\left(t_{j}\right)\right) \\
& \leq \lim _{j \rightarrow \infty}\left[-h\left(\left\|x_{t_{j}}\right\|\right)\right] \leq 0 .
\end{aligned}
$$

It implies $\lim _{j \rightarrow \infty}\left\|x\left(t_{j}\right)\right\|=0$ for each $i$, and thus leads to $\lim _{t \rightarrow \infty} x(t+\theta)=0$ for $\theta \in\left[-\tau_{i}, 0\right]$ which completes the proof.

Remark 1: In case of a time delay system without switching, i.e. $\sigma(t)=$ const., Theorem 3 reduces to the original Razumikhin theorem [11] (condition (iv) then follows from (iii) and is no longer needed). Furthermore, the values $\bar{V}_{i}\left(t_{j}, x_{t_{j}}\right), \bar{V}_{i}\left(t_{k}, x_{t_{k}}\right)$ at consecutive switching instants form a strictly decreasing sequence for each subsystem $i \in \mathcal{P}$. If the time delay is set to zero $\tau_{i}=0, i \in \mathcal{P}$, then the standard switched system is given and the stability result from [6] is recovered. For constant time delay, $\tau_{i}=$ const., a similar result as in [16] is achieved.

Remark 2: Theorem 3 is applicable to switched systems with an arbitrary finite number of subsystems. The subsystems can be nonlinear and time-varying.

Remark 3: In Theorem 3, the considered switched time delay system is described by an idealized mathematical delay differential equation containing controllers and plant. The state is assumed to have no state jump, and the initial condition $x_{t}$ for each time delay subsystem is well defined after switchings. However, for a real QoS controlled NCS as illustrated in Fig. 1, this assumption is no longer true as the following shows. Consider the plant $\dot{x}=f\left(x, u\left(t-\tau_{i}\right)\right)$ and the proportional state feedback controller $u(t)=K_{i} x(t)$. Controller and time delay are switched at time instant $t_{q}$ to $K_{j}$ and $\tau_{j}$, respectively. If $\tau_{i}>\tau_{j}$, then there is an overlap in the control signal $u$ arriving delayed at the plant over a time interval $\tau_{i}-\tau_{j}$. Accordingly, if $\tau_{i}<\tau_{j}$, the control signal remains undefined for the time interval $\tau_{j}-\tau_{i}$. This is equivalent to an overlapping or non-definition, resepectively, of the initial condition of subsystem $j$. For the sound definition of solutions, we treat the erratic definition of the initial condition as a kind of state jump, representing a degree of freedom during design. Here, for the overlapping case, we propose to drop the excrescent initial condition $x\left(t_{q}+\theta\right), \theta \in\left[-\tau_{i},-\left(\tau_{i}-\tau_{j}\right)\right]$. For the gap case, the absent initial condition is generated by holding the last received value, i.e. $\quad x\left(t_{q}+\theta\right)=x\left(t_{q}-\tau_{i}\right), \theta \in\left[-\tau_{j},-\left(\tau_{j}-\tau_{i}\right)\right] . \quad$ In both cases, the value $\bar{V}_{j}\left(t_{q}, x_{t_{q}}\right)$ is not changed, and thus the stability condition from Theorem 3 is not violated.

\section{Performance Evaluation}

In order to evaluate the potential of the proposed QoS control approach, simulations and experiments are conducted. Goal is to achieve a trade-off between control performance and network cost. The switching law should guarantee stability and optimal performance in the sense of this tradeoff. The generation of the switching law is discussed in the following.

\section{A. Towards Optimal Performance}

For high control performance of a NCS, generally, a low time delay is desirable, however high network costs are induced. The network cost-performace trade-off is formulated as multi-criteria optimization problem

$$
\min _{\sigma(t)} J=\int_{0}^{\infty} x(t)^{T} Q(t) x(t)+\eta_{\sigma(t)} d t,
$$

where $x(t)^{T} Q(t) x(t)$ is a measure for the control performance with $Q(t)$ a weighting matrix of appropriate dimension, and $\eta_{\sigma(t)}=\eta\left(\tau_{\sigma(t)}\right)$ represents the network cost associated with a certain time delay. The switching signal is generated by the optimizer representing the decision maker. The solution to this optimization problem gives the optimal switching instants and sequence. An analytical solution is difficult to find as the problem is hybrid, non-convex in general and contains delay differential equations. The further analysis is beyond the scope of this paper. Related optimal algorithms and conditions for switched systems are found in [17]-[21], however, without considering time delay in the subsystems. In order to demonstrate the benefit of QoS control, the optimization problem is solved offline in the following simulations and experiments with a similar approach as in [21]. The switching times and sequence are fixed a priori, the switching instants are determined by numerical optimization.

\section{B. Experimental System Model}

The position control of a one degree-of-freedom pendulum system, see Fig. 3, modelled as a damper-mass system

$$
u=J_{\text {sys }} \ddot{x}+b_{\text {sys }} \dot{x}
$$

is considered, where $x$ is the angular position, $u$ the motor torque. The inertia $J_{\text {sys }}=0.0274 \mathrm{kgm}^{2}$ and the damping $b_{\text {sys }}=0.2874 \mathrm{Nms} / \mathrm{rad}$ are identified from the experimental system via a least square method.

The position of the pendulum is fed back via the QoS communication channel switched between the two constant time delays: $\tau_{1}=15 \mathrm{~ms}$ and $\tau_{2}=6 \mathrm{~ms}$. The control torque is generated by PI controllers $u(t)=K_{I i} \int e(t) \mathrm{d} t+K_{P i} e(t)$, with $e=x_{d}-x$ the control error, and $i \in\{1,2\}$. They are switched synchronously with the time delay. With $\alpha=100$ 


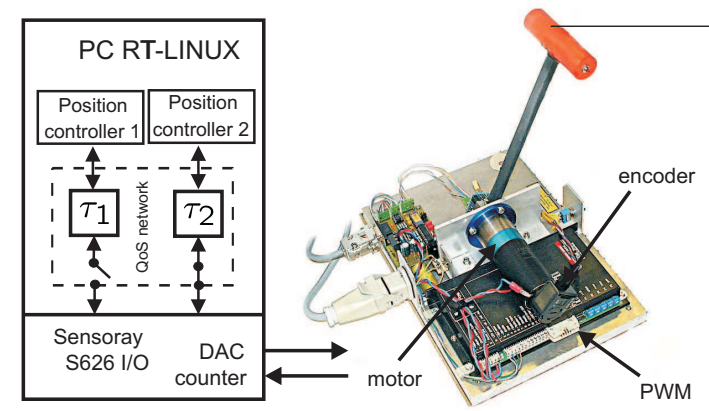

Fig. 3. Experimental one degree-of-freedom pendulum system.

and $\alpha_{1}=7.8$ the matrix inequality (6) gives the corresponding positive definite matrices $P_{i}$

$$
\begin{aligned}
& P_{1}=\left[\begin{array}{rrr}
0.0193 & -0.0163 & 0.0004 \\
-0.0163 & 0.0198 & -0.0018 \\
0.0004 & -0.0018 & 0.0017
\end{array}\right], \\
& P_{2}=\left[\begin{array}{rrr}
0.0184 & -0.0214 & -0.0004 \\
-0.0214 & 0.0346 & -0.0014 \\
-0.0004 & -0.0014 & 0.0026
\end{array}\right],
\end{aligned}
$$

using the lmi toolbox of MATLAB. The values of the PI controllers are $K_{P 1}=8.5, K_{I 1}=7$ for $\tau_{1}$ and $K_{P 2}=10, K_{I 2}=12$ for $\tau_{2}$, showing that for the low time delay mode 1 the controller can be tuned more aggressively.

\section{Simulations}

The simulation shows the effect of the switched time delay system with respect to stability and performance. Here the switching strategy is chosen such that the stability of system is guaranteed. The pendulum model is initiated with the high delay communication $\tau_{1}=15 \mathrm{~ms}$ along with an initial angle $x_{0}=1 \mathrm{rad}$; the reference input is $x_{d}=0 \mathrm{rad}$. The model is switched to low delay communication with $\tau_{2}=6 \mathrm{~ms}$ and the corresponding controller at $t=0.2 \mathrm{~s}$. The pendulum model is switched back and forth at $t=[0.8,1,1.2] \mathrm{s}$ and stays at high delay communication from $t=1.2 \mathrm{~s}$ to the end. The evolution of the systems state $x$ is shown in Fig. 4 (b) by the solid line. For comparison the state evolution with high delay communication and low delay communication are depicted by the dash-dotted and dashed line in Fig. 4 (b), respectively. Even for this rather arbitrary switching strategy, the performance in terms of overshoot and settling time of the switched time delay system is better than the system only with high delay communication. The evolution of the piecewise continuous Lyapunov function of the switched system is shown in Fig. 4 (c). The values at the consecutive switching instants form a strictly decreasing sequence, the switched time delay system is asymptotically stable.

\section{Experiments}

The experimental validation is performed using the same controller and time delays as in the simulation. The experimental testbed consists of the $1 \mathrm{DOF}$ pendulum as shown in Fig. 3 connected to a PC running under RT Linux. The
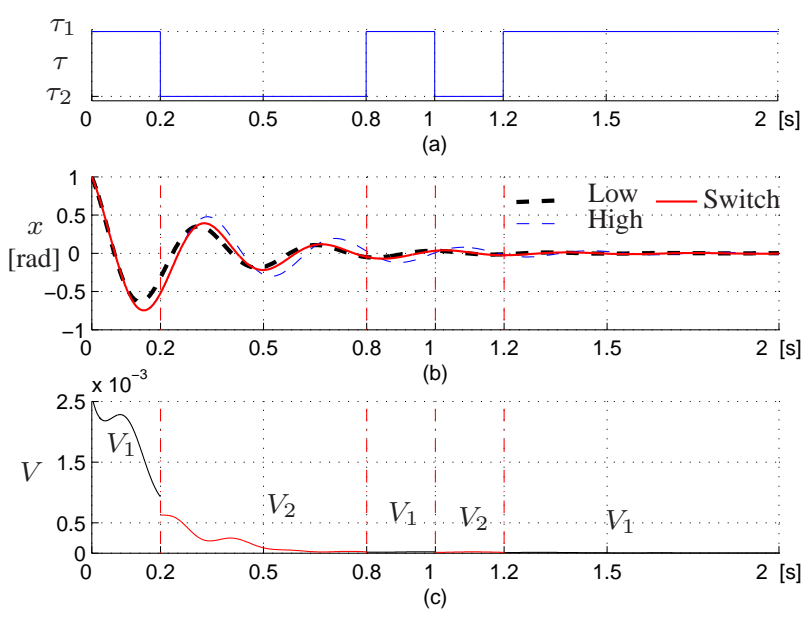

Fig. 4. Simulation results: Time delay evolution (a), state evolution (b) and piecewise continuous Lyapunov function (b) of the pendulum system, $V_{1}$ and $V_{2}$ denote the Lyapunov functions corresponding to the subsystems 1 and 2 with the time delays $\tau_{1}=15 \mathrm{~ms}$ and $\tau_{2}=6 \mathrm{~ms}$.

original design of the pendulum can be found in [22]. The DC-motor current, resulting in torque, is provided by the PWM amplifier operated under current control. The reference signal is given by voltage from the D/A converter and is an output of the I/O board. The position of the lever is measured by an optic pulse incremental encoder and processed by a quadrature encoder on the I/O board. The control loop including the controller, the time delays and the switching strategy are implemented in MATLAB/SIMULINK blocksets. Standalone realtime code is generated directly from the SIMULINK model. All the experiments are performed with a sampling time interval of $T_{A}=0.001 \mathrm{~s}$.

The switching instants are determined using the optimization procedure proposed in Section V-A. The network costs are heuristically set to $\eta\left(\tau_{1}\right)=2 \times 10^{-5} \mathrm{~s}^{-1}$ and $\eta\left(\tau_{2}\right)=5 \times 10^{-5} \mathrm{~s}^{-1}$. The initial conditions are specified by $x_{0}^{T}=[0,0], \theta \in\left[-\tau_{1}, 0\right]$ and $\sigma(0)=1$, i.e. subsystem 1 with time delay $\tau_{1}$. A step function, which has the amplitude $0.1 \mathrm{rad}$ at $t=1 \mathrm{~s}$ serves as position reference input $x_{d}$ to the system. The position error is used for the optimization in the cost functional (7), the weight is set to $Q(t)=I$. The number $k$ of switches and the switching order are fixed prior to optimization, here with $k=2$ and $\left.\left\{\left(t_{1}, 2\right),\left(t_{2}, 1\right)\right)\right\}$, i.e. switching into subsystem 2 takes place at $t=t_{1}$. The optimization problem is solved offline using the fminsearch algorithm from the MATLAB optimization toolbox resulting in the switching times $t_{1}=1 \mathrm{~s}$ and $t_{2}=1.5 \mathrm{~s}$, respectively.

The evolution of the time delay is presented in Fig. 5 (a). The position evolution for the switching approach, and for comparison also for low and high time delay without switching, is presented Fig. 5 (b). Surprisingly, the switched system shows a control performance comparable to the system without switching and only with low time delay, see also Table I. However, the network cost of the switched 


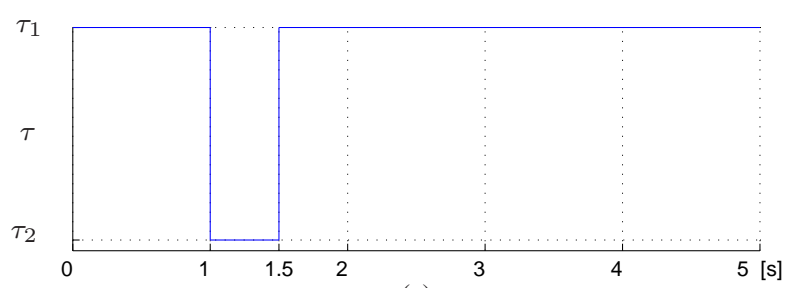

(a)

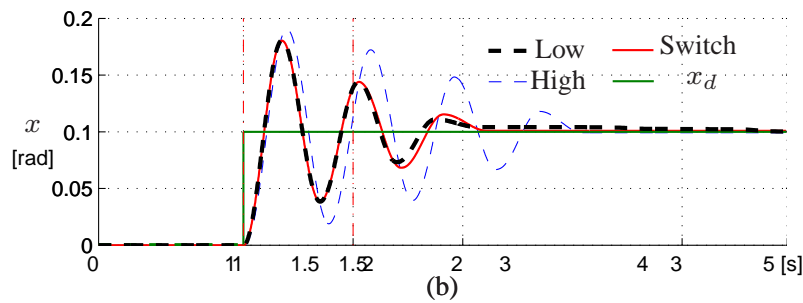

Fig. 5. Experimental results: Communication time delay (a) and position evolution (b) of the experimental pendulum system.

TABLE I: EXPERIMENTAL RESULTS

\begin{tabular}{|l|ccc|}
\hline & High delay & Low delay & Switched delay \\
\hline Settling time $^{1}[\mathrm{~s}]$ & 1.153 & $1.061^{2}$ & 1.033 \\
Overshoot [\%] & 90.5 & 80.7 & 80.4 \\
Network costs & $10 \times 10^{-5}$ & $25 \times 10^{-5}$ & $12.28 \times 10^{-5}$ \\
\hline
\end{tabular}

time delay system is $50.88 \%$ lower. Less overshoot and a $10.14 \%$ lower settling time than with high time delay only, is observed. These experimental results show the potential of proposed approach and are indeed very promising for a further investigation of QoS control in NCS. For real application a number of challenges remain to be addressed, e.g. online generation of a (sub-)optimal switching law and consideration of time delay in the switching signal.

\section{CONCLUSION}

In this paper a novel concept of Quality-of-Service control for networked control system (NCS) is introduced. The NCS itself and the communication quality in terms of time delay is conjointly controlled to meet control performance and network cost objectives. The time delay of the communication network is switched together with the controller resulting in switched system with piecewise constant time delay. A sufficient stability condition is derived based on the Razumikhin approach for time delay systems and the piecewise continuous Lyapunov function method for switched systems. Simulations and experiments show that the proposed QoS control approach is very promising as a trade-off between control performance and network cost is achieved.

Future research includes the extension of the results to the less conservative Lyapunov-Krasovskii approach and online generation of the switching law.

${ }^{1}$ The settling time is defined for the output response first to reach and thereafter remain within $5 \%$ of the final value.

${ }^{2}$ Theoretically, the settling time for low time delay without switching is expected to be lower than with switched time delay. A possible explanation for the slightly higher settling time are nonlinear phenomena such as backlash and friction.

\section{ACKNOWLEDGMENT}

The valuable and helpful comments by the anonymous reviewers are highly appreciated.

\section{REFERENCES}

[1] S. Shenker and J. Wroclawski, "General Characterization Parameters for Integrated Service Network Elements," IETF RFC 2215, 1997.

[2] S. Petersson, Analysis and Design of Hybrid Systems. PhD thesis, Chalmers University of Technology, Göteborg, Sweden, 1999.

[3] D. Liberzon and A. S. Morse, "Basic problems in stability and design of switched systems," IEEE Control Systems Magazine, vol. 19, pp. 59-70, October 1999.

[4] P. Peleties and R. DeCarlo, "Asymptotic stability of m-switched systems using lyapunov functions," in Proceedings of the $31^{\text {th }}$ Conference on Decision and Control, (Tucson, Artzons), pp. 3438-3489, 1992.

[5] R. A. Decarlo, M. S. Branicky, S. Petersson, and B. Lennartson, "Perspectives and results on the stability and stabilization of hybrid systems," in Proceeding of the IEEE, vol. 88.

[6] H. Ye, N. Michel, and L. Hou, "Stability theory for hybrid dynamical systems," IEEE Transactions on Automatic Control, vol. 43, pp. 461474, April 1998.

[7] A. S. Morse, "Supervisory control of families of linear set-point controllers - part 1: exact matching," IEEE Transactions on Automatic Control, vol. 41, pp. 1413-1431, October 1996.

[8] J. Hespanha and A. Morse, "Stability of switched systems with average dwell-time." Technical report, EE-Systems, University of Southern California/ Lab. for Control Science and Eng., Yale University, 1999.

[9] J.-P. Richard, "Time-delay systems: an overview of some recent advances and open problems," automatica, vol. 39, pp. 1667-1694, 2003.

[10] V. L. Kharitonov, "Robust stability analysis of time delay systems: A survey," Annual Reviews in Control, vol. 23, pp. 185-196, 1999.

[11] J. K. Hale and S. M. V. Lunel, Introduction to Functional Differential Equations. Springer-Verlag, 1993.

[12] K. Gu, V. L. Kharitonov, and J. Chen, Stability of Time-Delay Systems. Birkhäuser, 2003.

[13] Q.-Y. Tong, G.-F. Yan, and G.-Z. Zhao, "Stability analysis of hybrid systems with time-varing delayed perturbation via single lyapunov function," in Proceedings of the Second International Conference on Machine Learning and Cybernetics, pp. 919-922, 2003.

[14] G. Zheng, M. Tan, and Y. Song, "An approach to analyze the stability of a class of hybrid systems wiht delay," in Proceeding of $5^{\text {th }}$ World Congress on Intelligent Contorl and Automation, (Hangzhou, P.R. China).

[15] P. Yan and H. Özbay, "Stability analysis of switched time-delay systems," in Proceedings of the 16th IFAC World Congress, (Prague, Czech Republic), 2005.

[16] R. Yuan, Z. Jing, and L. Chen, "Uniform asymptotic stability of hybrid dynamical systems with delay," IEEE Transactions on Automatic Control, vol. 48, pp. 344-348, February 2003.

[17] M. Egerstedt, Y. Wardi, and F. Delmotte, "Optimal control of switched dynamical systems," in Proceedings of the 42nd IEEE Conference on Decision and Control, (Maui, Hawaii USA), 2003.

[18] M. S. Shaikh and P. E. Caines, "On the optimal control of hybrid systems: Optimization of switching times and combinatoric location schedules," in Proceedings of the American Control Conference, (Denver, Colorado), 2003.

[19] B. Lincoln and B. Bernhardsson, "Lqr optimization of linear system switching," IEEE Transactions on Automatic Control, vol. 47, pp. 1701-1705, October 2002.

[20] R. Luus and Y.-Q. Chen, "Optimal switching control via direct search optimization," in Proceedings of the 2003 IEEE International Symposium on Intelligent Control, (Houston, Texa USA), pp. 371-376, 2003.

[21] X. Xu and P. J. Antsaklis, "An approach for solving general switched linear quadratic optimal control problems," in Proceedings of the $40^{\text {th }}$ Conference on Decision and Control, (Orlando, Florida USA), pp. 2478-2483, 2001.

[22] H. Baier, M. Buss, F. Freyberger, J. Hoogen, P. Kammermeier, and G. Schmidt, "Distributed pc-based haptic, visual and acoustic telepresence system-experiments in virtual and remote environments," in Proceedings of the IEEE Virtual Reality Conference VR'99, (Houston, TX), 1999. 